-This is a non-peer reviewed preprint submitted to EarthArXiv-

\title{
1 LPDynR: a new tool to calculate the Land Productivity
}

\section{Dynamics indicator}

$5{ }^{1}$ ASTER Projects. Barri Reboll, 9, 1r. 08694 Guardiola de Berguedà (Barcelona), SPAIN

$6{ }^{2}$ European Environment Agency. Geospatial Information Services Group. Copenhagen,

7 DENMARK

$8{ }^{3}$ European Commission - Joint Research Centre (JRC). Directorate D - Sustainable

9 Resources. Unit D6 - Knowledge for Sustainable Development \& Food Security Unit.

10 Via Enrico Fermi 2749. I-21027 Ispra (VA), ITALY

$11 \square$ Correspondence: Michael Cherlet <michael.cherlet@ec.europa.eu>

15 Keywords: Land Productivity, Ecosystem Dynamics, Land Degradation, Desertification,

16 Vegetation 
-This is a non-peer reviewed preprint submitted to EarthArXiv-

\section{Abstract}

19 As part of the UN Sustainable Development Goal 15 (Life on Land), the indicator 15.3.1

20 is adopted to measure the Land Degradation Neutrality. Land Degradation Neutrality is 21 addressed as stable — or increasing — state in the amount and quality of land resources

22 required to support ecosystem functions and services and enhance food security during a 23 certain period of time. It is a binary indicator (i.e. degraded/not degraded), expressed as 24 the proportion of land that is degraded over total land area within each land type, and is

25 based on three sub-indicators: (1) Trends in Land Cover, (2) Land Productivity and (3)

26 Carbon Stocks.

27 The Land Productivity sub-indicator (LP) refers to the total above-ground Net Primary

28 Production and reflects changes in health and productive capacity of the land. Declining

29 trends interpreted with ancillary data such as e.g. information on non-adapted agricultural

30 practices possibly combined with low income can be usually understood as land

31 degradation. LP can be calculated using the Land Productivity Dynamics (LPD)

32 approach, which is the methodological basis of the R-based tool LPDynR presented in

33 this article. It uses vegetation-related indices (phenology and productivity) derived from

34 time series of remote sensed vegetation indices to estimate ecosystem dynamics and

35 change. The final result of the LPD indicator is a categorical map with 5 classes of land

36 productivity dynamics, ranging from declining to increasing productivity. As an example

37 of $L P D y n R$ functionalities, we present a case study for Europe. 
-This is a non-peer reviewed preprint submitted to EarthArXiv-

\section{$39 \quad 1$ Introduction}

40 The United Nations General Assembly designed in 2015 a collection of 17 global goals,

41 so called Sustainable Development Goals (SDGs; UN, 2015), with the general aim of

42 "achieving a better and more sustainable future for all", and which are intended to be

43 accomplished by 2030 . Each SDG is subdivided into a list of targets which, in turn, go

44 together with indicators to be able to measure their progress and success. Such indicators

45 have to be credible, based on standardized methodologies and, often, have to be spatially

46 explicit (Dubovyk, 2017).

47 The SDG-15, entitled Life on Land, has among its targets the 15.3, which expects "to

48 combat desertification, restore degraded land and soil, including land affected by

49 desertification, drought and floods, and strive to achieve a land degradation-neutral

50 world". In this context, Land Degradation Neutrality (LDN) is defined as the stable (or

51 increasing) state regarding the amount and quality of land resources required to support

52 ecosystem functions and services and enhance food security during a certain period of

53 time (UNCCD, 2015).

54 The indicator 15.3.1 is adopted to measure the LDN and is expressed as the proportion of

55 land that is degraded over total land area. It is a binary indicator (i.e. degraded/not

56 degraded) based on three sub-indicators calculated separately: (1) Trends in Land Cover,

57 (2) Land Productivity and (3) Carbon Stocks (Sims et al., 2020, 2017). While the first

58 two can capture relatively fast changes, carbon stocks reflect slower changes which

59 suggest a longer-term trajectory (Orr et al., 2017). Following a "one-out-all-out" process,

60 the indicator identifies an area as degraded if one of the sub-indicators shows 
-This is a non-peer reviewed preprint submitted to EarthArXiv-

61 degradation. The three sub-indicators must be comparable among territories and based on

62 standardized sources and methods. The data can be collected through existing sources,

63 such as maps, reports or databases, but also can be derived from Earth observation (EO)

64 imagery using remote sensing tools.

65 The Land Productivity sub-indicator (LP), addressed in this document, approximates the

66 total above-ground net primary productivity (NPP), which can be defined as the total

67 energy fixed by plants minus their respiration. Such energy is transformed into biomass

68 which, in turn, allows ecosystems to develop their functions and deliver essential

69 services. Therefore, LP reflects changes in health and productive capacity of the land and

70 its declining trends can be usually understood as land degradation (Cherlet et al., 2018;

71 Prince, 2009; Yengoh et al., 2015). The World Atlas of Desertification (Cherlet et al.,

72 2018) suggests that the LP sub-indicator can be calculated using the Land Productivity

73 Dynamics (LPD) approach. LPD was first developed by Ivits and Cherlet (2013) and is

74 the methodological basis of the LPDynR tool presented in this article.

\section{Land Productivity Dynamics and LPDynR}

76 The Land Productivity Dynamics (LPD) approach is based fundamentally on the use of

77 time series of vegetation-related indices derived from remote sensed imagery, such as the

78 normalized difference vegetation index (NDVI) or the plant phenology index (PPI).

79 NDVI, for example, can be used as a proxy for land productivity, as many studies at

80 global and local scales have identified a strong relationship between NDVI and NPP

81 (Ivits and Cherlet, 2013; Prince, 2009; Yengoh et al., 2015, and references therein). The

82 LPD approach often uses phenological and productivity-re lated variables derived from 
-This is a non-peer reviewed preprint submitted to EarthArXiv-

83 time series of NDVI, given that these can provide additional information on several

84 aspects of vegetation/land cover functional composition in relation to ecosystem

85 dynamics and change (E. Ivits, M. Cherlet, Mehl, et al., 2013). These dynamics of the

86 ecosystems, which might eventually drive land degradation, can be caused by human

87 activities and/or biophysical processes, as well as other processes indirectly tied to them,

88 such as climate change (Yengoh et al., 2015). While the most commonly used

89 phenological parameters are the beginning and the end date of the vegetation growing

90 season, together with the season length in number of days, the ones related to land

91 productivity are e.g. accumulations of vegetation index values over time, mostly during

92 the growing season as defined by the season start and end date. These approximate NPP

93 within the growing season.

94 The final result of the LPD indicator is a categorical map with 5 classes of land

95 productivity dynamics, ranging from declining to increasing productivity over a target

96 time period. It is the result of a combined assessment of two sources of information, as

97 seen in Figure 1. The first layer is the Long-Term Change Map. In general terms, it

98 shows the tendency of change of land productivity (positive or negative) and the effect on

99 productivity levels that this tendency might have had on a particular original point after a

100 certain period of time. The second layer is the Current Status Map, which provides

101 information on the current levels of land productivity in relation to its potential, being

102 current the end of the target time period. It compares the local productivity with the range

103 of productivity across similar areas in terms of land cover or bioclimatic traits (Sims et

104 al., 2017). Further explanations for both branches will be given in the respective sections

105 below. 
-This is a non-peer reviewed preprint submitted to EarthArXiv-

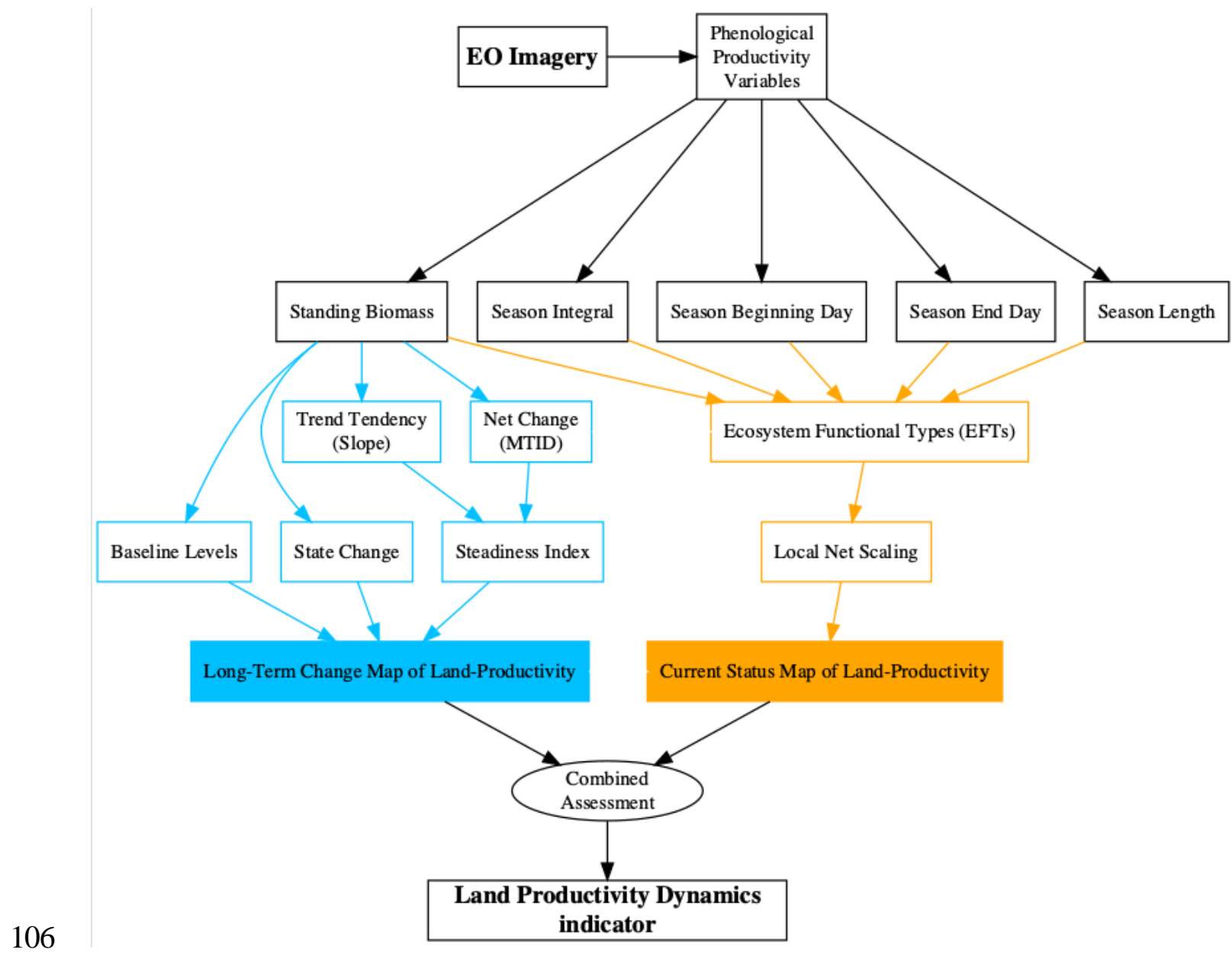

107 Figure 1: Flowchart of the process to calculate the Land Productivity Dynamics indicator and used by LPDynR

110 Following the LPD approach, $L P D y n R$ is an R-based tool (i.e. an R package) which

111 allows the user to produce the final Land Productivity Dynamics Map using as inputs a

112 set of time series of phenological and/or productivity variables (multi-band GeoTIFF

113 rasters). By means of the different functions included in the package, it produces

114 intermediate layers (e.g. Steadiness Index, Ecosystem Functional Types; see Figure 1)

115 which are used to calculate both the Long-Term Change Map and the Current Status 
-This is a non-peer reviewed preprint submitted to EarthArXiv-

116 Map. In addition, several parameters can be set along the process in order to reflect the

117 preferences of the user. The functions included in the package have no limitations

118 regarding the number of years included in the time series, the variables to use or the

119 spatial extent and resolution. While LPDynR v1.0.1 can be installed from CRAN

120 (https://CRAN.R-project.org/package=LPDynR), the latest version is available at

121 https://github.com/xavi-rp/LPDynR.

\section{Data set preparation}

123 A case study is presented in order to illustrate the methodology implemented in the

$124 L P D y n R$ package to calculate the LPD indicator. In this case, a data set of 5 phenological

125 and productivity-related variables were used, at European level and on a $0.5 \mathrm{~km}$ of spatial

126 resolution, produced by the European Environment Agency - European Commission

127 (EEA). They are all derived from time series (2000-2019) of MODIS imagery and its

128 derived product Plant Phenology Index (PPI; Jin and Eklundh, 2014). PPI is linearly

129 related to the canopy green leaf area index (LAI) and has a temporal pattern very similar

130 to the one shown by the gross primary productivity (GPP) estimated by flux towers at

131 ground reference stations. The five variables are produced using the software TIMESAT

132 (Jönsson and Eklundh, 2004). At the moment of writing this article, these time series are

133 not yet published, however more information about the previous freely distributed data

134 set (2000-2016) by the EEA can be found in their website

135 (https://sdi.eea.europa.eu/catalogue/srv/eng/catalog.search\#/home). For example, the

136 details for above ground vegetation productivity can be found in 
-This is a non-peer reviewed preprint submitted to EarthArXiv-

137 https://sdi.eea.europa.eu/catalogue/srv/eng/catalog.search\#/metadata/29ae2d47-7af2-

138 4c09-ba5f-e2fbb7c2b0d1. The five variables used were:

139 - Above ground vegetation productivity (from now on, SB)

140 - Above ground season vegetation productivity (from now on, CF)

141 - Start of vegetation growing season (from now on, SBD)

142 - End of vegetation growing season (from now on, SED)

143 - Vegetation growing season length (from now on, SL)

144 In the LPDynR v.1.0.1, the functions use multi-band GeoTIFF rasters to start the process,

145 one per phenological/productivity variable. Each band of each raster contains one of the

146 years of the time series.

147 It is also important to note that LPDynR comes with a sample data set, which can be used

148 to run tests, as well as some examples in the form of "vignettes" attached to the package.

\section{Long Term Change Map of land productivity}

150 As seen in Figure 1 and explained above, the Land Productivity Dynamics indicator is

151 produced by combining two input layers. The first layer is the Long-Term Change Map

152 (also called "tendency map"). The tendency layer combines information on the trend of

153 land productivity dynamics (positive or negative), the level of productivity of the

154 ecosystem at the start of the time series, as well as whether it has changed its productivity

155 state or not in the period under study (Ivits and Cherlet, 2013). Using such multi-source

156 information for the Long-Term Change Map instead of a trend significance assessment 
-This is a non-peer reviewed preprint submitted to EarthArXiv-

157 was chosen to better describe the state and change of ecosystems. For instance, even

158 though vegetation development presents a long-term negative dynamics (e.g. negative

159 slope of a linear trend), the negative trend might not be strong enough to decrease the

160 level of productivity such that the starting productivity state changes drastically. This

161 could result to be a non-significant trend in linear trend analysis leaving the pixel out for

162 further analysis which is not wishful in the land degradation analysis. The way in which

163 the three sources of information are calculated for the Long Term Change Map using a

164 land productivity variable is described in the following subsections.

\section{4.1 Steadiness Index}

166 The first of the three metrics which integrates the Long-Term Change Map represents the

167 long-term tendency of change of the natural systems, being either positive or negative.

168 This metrics is the Steadiness Index (Ivits, Cherlet, Sommer, et al., 2013) and can be

169 calculated using the function steadiness(). The Steadiness Index is based on the

170 combination of two other metrics which are calculated per pixel by the same function: (1)

171 the slope derived from fitting a linear trend on the time series and (2) the net change of

172 the productivity level of the same period.

173 The use of a linear regression would imply to respect the linear trend results by strict

174 statistical assumptions for confidence intervals and significance tests, such as

175 heteroscedasticity, normal distribution of the errors, no autocorrelation between the

176 observations and a deterministic process. Most often, these assumptions are not

177 accomplished when working with time series of remote sensed products, and the use of

178 non-parametric trend measures are not adequate either (Ivits, Cherlet, Sommer, et al., 
-This is a non-peer reviewed preprint submitted to EarthArXiv-

179 2013). This is why the Steadiness Index only keeps classes of tendency and no more tests

180 are run for assessing its significance. Therefore, only the sign (positive or negative) of the

181 slope of the trend is kept as the value of each pixel's tendency of ecosystem dynamics. In

182 addition, the net change of the productivity variable, in the units of the applied vegetation

183 index, is calculated for the same time window and per pixel using the Multi-Temporal

184 Image Differencing method (MTID; Guo et al., 2008). Afterwards, MTID is also

185 transformed into positive or negative net change. Finally, the two classes of both metrics

186 (slope of the linear function and net change category) are combined into four "steadiness"

187 categories as seen in Table 1. Figure 2A represents the 4-class map of the Steadiness

188 Index for the case study.

189 Table 1: Description of the four Steadiness Index classes and how they are derived based on the 190 combination of the signs of both the slope of the linear function and the net change

\begin{tabular}{cccc} 
Steadiness Class & Slope & Net Change & Description \\
\hline Steadiness 1 & - & - & $\begin{array}{c}\text { Strong negative ecosystem dynamics } \\
\text { (possibility changing equilibrium) }\end{array}$ \\
Steadiness2 & - & + & $\begin{array}{c}\text { Moderate negative ecosystem dynamics } \\
\text { (likely remain in current equilibrium) }\end{array}$ \\
Steadiness3 & + & - & $\begin{array}{c}\text { Moderate positive ecosystem dynamic s } \\
\text { (likely remain in current equilibrium) }\end{array}$ \\
Steadiness4 & + & + & $\begin{array}{c}\text { Strong positive ecosystem dynamics } \\
\text { (possibility changing equilibrium) }\end{array}$
\end{tabular}


-This is a non-peer reviewed preprint submitted to EarthArXiv-

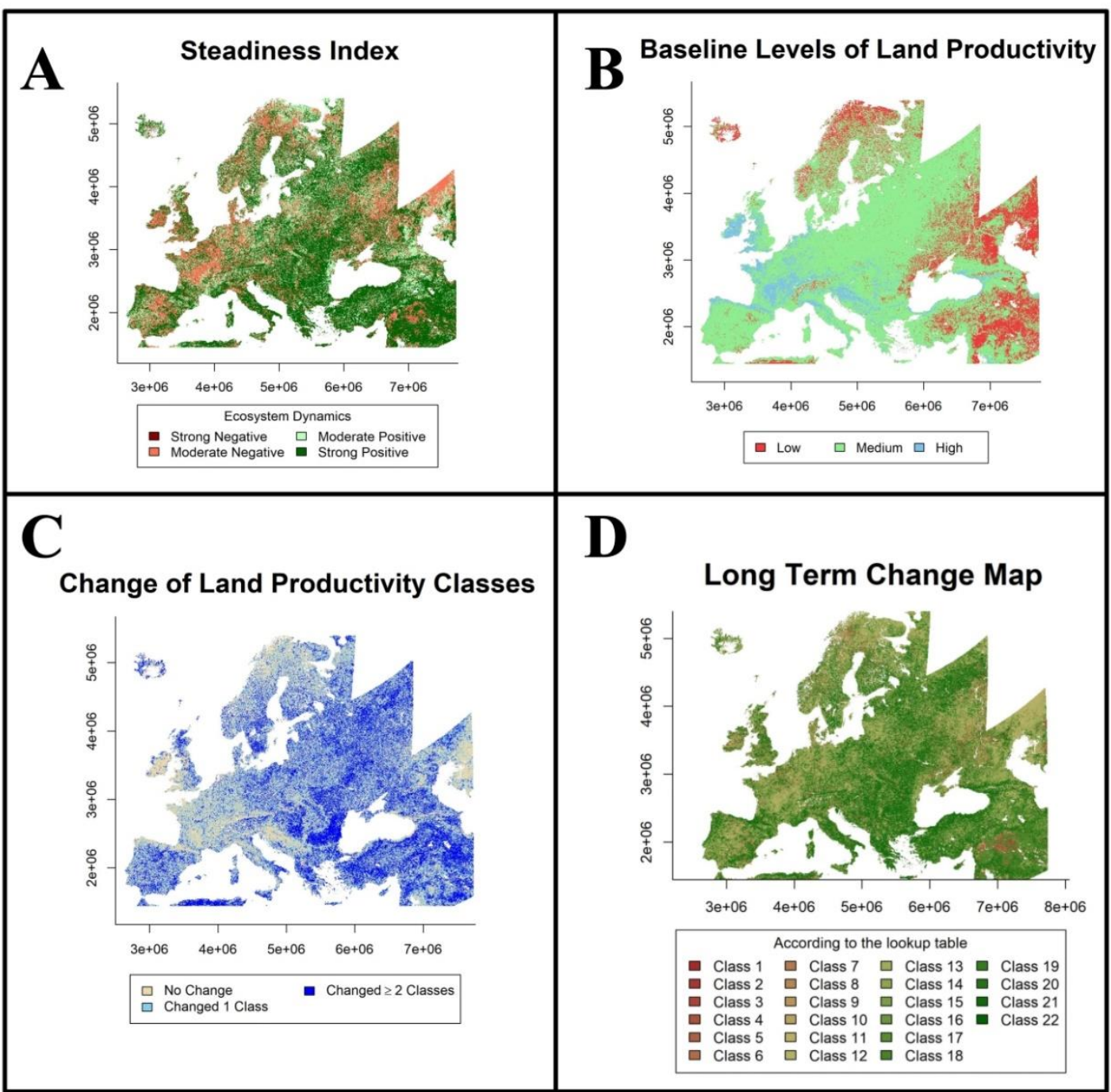

192

193 Figure 2: (A) Steadiness Index, (B) baseline levels and (C) state change maps for the case study

194 based on the 'Above ground vegetation productivity'variable. (D) Land productivity Long Term

195 Change Map for the case study based on the combination of the previous three maps. 
-This is a non-peer reviewed preprint submitted to EarthArXiv-

\section{$198 \quad 4.2$ Baseline levels of productivity}

199 The second source of information for the derivation of the Long-Term Change Map is the

200 baseline levels of the productivity variable at the beginning of the time series.

201 For the calculation of the baseline levels of land productivity at the beginning of the time

202 series, LPDynR categorizes productivity values into three classes: low, medium and high.

203 To do that, the function baseline_lev() averages the first $n$ years of the time series in

204 order to avoid extreme events, such as abnormal droughts in wet areas, etc, which would

205 skew the distribution of productivity values into too high or low values. The number of

206 years to be considered by the average function can be set by passing the argument

207 yearsBaseline to the function. The default value is 3 years; averaging more years would

208 move the baseline value closer to the mean of the time series, which would not describe

209 the baseline anymore.

210 After the average of the $n$ number of years is calculated, baseline_lev() first classifies the

211 pixels into 10 classes using 10-quantiles equalling to the corresponding percentile levels.

212 The reason for this intermediate step is that, if directly opted for three classes (i.e. low,

213 medium and high), the number of pixels per category would be classified homogeneously

214 (i.e. $33.3 \%$ of pixels/class), which is a statistically correct but an over simplified

215 representation of baseline status. Instead, $L P D y n R$ allows the user to define the percentile

216 level to be used based on local knowledge. For example in dryland ecosystems or in

217 boreal regions different average productivity level can be defined as low, medium or high

218 values. The United Nations Development Programme (UNPD, https://www.undp.org) for

219 example declares that $40 \%$ of the World's land resources are drylands (Middleton et al., 
-This is a non-peer reviewed preprint submitted to EarthArXiv-

220 2011), while the World Atlas of Desertification updated this proportion to $37.2 \%$ (Cherlet

221 et al., 2018). Therefore, in global applications one might choose $37.2 \%$ of pixels to be

222 classified as "low level" of productivity. Consequently, as default, the global application

223 of $L P D y n R$ classifies the first four groups of pixels, i.e. 40 percentile (after rounding

$22437.2 \%$ ), as "low" baseline productivity level, the five consecutive groups between 50 and

22590 percentile as "medium" productivity level and the rest $10 \%$ of pixels with the highest

226 average productivity levels, as "high" baseline. Both the proportion of pixels classified as

227 low level and high level of land productivity can be set by passing to baseline_lev() the

228 arguments drylandProp and highprodProp, respectively. The function classifies the rest

229 of the pixels ((100 - (drylandProp + highprodProp $))$ as medium level. The assumption of

230 classifying $40 \%$ of pixels as low productive is valid at global level, however, the

231 proportion of drylands/low level of productivity should be modified for local and regional

232 studies. For example, at the European level, drylands cover $20 \%$ of total land (FAO,

233 2019). This proportion has been used in the case study and the resulting 3-class map

234 showing the estimation of levels of productivity at the beginning of the time series can be

235 seen in Figure 2B.

$236 \quad 4.3$ Change of state of productivity

237 The third layer used for the land productivity Long-Term Change Map is the change of

238 the state of the productivity level during the time window under study. This aspect is

239 necessary for land degradation assessments as it reports whether pre-set productivity state

240 thresholds have been surpassed or not, which can be a consequence of either the natural 
-This is a non-peer reviewed preprint submitted to EarthArXiv-

241 resilience, new land use/practices that have been introduced, or impacts of other

242 manmade or natural phenomena (Ivits and Cherlet, 2013).

243 To calculate the state change per pixel, the function state_change() uses both the

244 productivity baseline level at the beginning of the time series, as described in the

245 previous subsection, and the productivity state level at the end of the time series. This

246 final state is calculated in the same way as the baseline level, i.e. (1) averaging the last 3

247 years and (2) classifying into 10 categories using 10-quantiles. The reason for using a 10-

248 class classification is that it would be difficult to approximate if the change of one state to

249 another was due to a big or a small change. Instead, using the 10-class classification for

250 the final productivity state, one can address if a pixel has moved from class 5 to 4 (small

251 change) or from class 9 to 4 (big change).

252 Once the class change per pixel has been calculated, either with positive or negative

253 results, the map is categorized into 3 final classes: (1) no change, (2) changed between 1

254 and $x$ classes or (3) changed more than $x$ classes, where $x$ can be defined by the user by

255 passing the argument changeNclass to the function (default is 1). See Figure $2 \mathrm{C}$ for a

256 map of the state change in the case study.

\section{4.4 Long Term Change Map}

258 The land productivity Long-Term Change Map is one of the two pillars of the LPD

259 indicator (Figure 1) calculated with LPDynR. This map is calculated by the combination

260 of the Steadiness Index, the productivity levels at the beginning of the time series and the

261 change of the state of productivity between the beginning and the end of the time series. 
-This is a non-peer reviewed preprint submitted to EarthArXiv-

262 The function LongTermChange() performs the combination of the three qualitative

263 metrics mentioned before into the Long-Term Change Map, resulting in 22 new

264 categories as shown in Table 2. The resulting map for the case study is presented in

265 Figure 2D.

266

267

Table 2: Lookup table for the land productivity Long Term Change Map (Steadiness Index + 268 BaseLine Levels + State Change)

Change of productivity at the end of the time series

No Change Changed 1 to $x \quad$ Changed $>x$
classes classes

\section{Steadiness Index / Baseline productivity}

$\begin{array}{rccc}\text { St1 low } & 1 & 2 & 3 \\ \text { St1 med. } & 4 & 5 & 6 \\ \text { St1 high } & 7 & 8 & 9 \\ \text { St2 low } & 10 & 10 & 10 \\ \text { St2 med. } & 11 & 11 & 11 \\ \text { St2 high } & 12 & 12 & 12 \\ \text { St3 low } & 13 & 13 & 13 \\ \text { St3 med. } & 14 & 14 & 14 \\ \text { St3 high } & 15 & 15 & 15 \\ \text { St4 low } & 16 & 17 & 18 \\ \text { St4 med. } & 19 & 20 & 21 \\ \text { St4 high } & 22 & 22 & 22\end{array}$

270 At this point, the user might want to finalise the LPD calculation avoiding the second part

271 of the methodology proposed by Ivits and Cherlet (2013), which is the Current Status

272 Map of Land Productivity. To do this, the function LPD_CombAssess (see further 
-This is a non-peer reviewed preprint submitted to EarthArXiv-

273 explanations in the respective subsection below) can be called to reclassify the 22-class

274 Long-Term Change Map into the final 5 classes of LPD.

2755 Current Status Map of land productivity

276 The Land Productivity Dynamics indicator is composed of two base layers: the Long-

277 Term Change Map of Land Productivity and the Current Status Map of Land Productivity

278 (as shown in Figure 1). After the long-term productivity dynamics described previously

279 (i.e. Long-Term Change Map) is calculated, the second source of information needed is

280 the current level of land productivity. For this purpose, a Local Net Scaling approach is

281 implemented (Prince, 2009). Such approach estimates the level of land productivity of

282 each pixel relative to its neighbours with similar characteristics of their land functions. In

283 other words, it calculates the potential level of productivity of each pixel within a

284 homogeneous land unit. The Current Status Map may help, for instance, to identify areas

285 which, although having a positive trend of productivity over time, their levels of current

286 productivity are low relative to the pixels in the same homogeneous land unit and, thus,

287 they might be still suffering land degradation (Sims et al., 2017). A first step for the

288 calculation of the Current Status Map, therefore, is the derivation of the homogeneous

289 land units across the area of study.

$290 \quad 5.1$ Ecosystem Functional Types (EFTs)

291 The methodology implemented in LPDynR to derive homogeneous land units, or

292 Ecosystem Functional Types (EFTs), is adapted from Ivits, Cherlet, Horion et al. (2013).

293 It is basically a clustering process which uses, in this case, phenological and productivity 
-This is a non-peer reviewed preprint submitted to EarthArXiv-

294 variables to create the ecosystem functional groups. Among the different unsupervised

295 clustering techniques available for data grouping, K-means has been chosen. K-means is

296 widely used in data science mainly due to its relative simplicity of implementation and

297 interpretation.

298 Originally, the unsupervised classification was performed after a three-steps pre-

299 processing of the phenology and productivity variables (see Chapter 3, Dataset

300 preparation): (1) removing highly correlated variables to avoid multicollinearity; (2) a

301 first Principal Component Analysis (PCA) to select the optimal number of PCs and their

302 associated variables showing the highest loadings; and (3) a final PCA to clearly

303 associate each PC with one variable. However, test runs in this study (see Supplementary

304 Material S1) have shown that the final LPD indicator does not differ significantly when it

305 is derived using the raw phenological/productivity variables. Therefore, although the two-

306 PCAs step is also implemented in LPDynR, only the removing of highly correlated

307 variables (e.g. $|r|>0.7$ ) is recommended before running the k-means clustering.

308 In order to check for multicollinearity among the variables, the function rm_multicol()

309 first calculates their averages among the years of the time series. Then, the process

310 internally runs the function removeCollinearity() from the package virtualspecies (Leroy

311 et al., 2016). This function allows the user to set up the minimum Pearson's correlation

312 absolute value, which can be modified by passing the argument multicol_cutoff. It is

313 established to be $r=0.7$ as default. A subset of random points of the data set can be used

314 for the calculation of the correlation coefficient in case the rasters have a large number of

315 pixels and the user wants to speed up the process. The default number of randomly

316 selected points is $10 \%$ of total pixels in the raster. However, the number of points can be 
-This is a non-peer reviewed preprint submitted to EarthArXiv-

317 selected by passing sample.points $=$ FALSE and nb.points equal to the required amount

318 of points. Finally, the function automatically creates a multi band raster where each band

319 corresponds to one randomly selected variable of each group of correlation. In addition, a

320 dendrogram to visualize the groups of intercorrelated variables can be plotted if the user

321 wants to, although not by default. For the present case study, which was run with five

322 variables, the dendrogram produced can be seen in Supplementary Material S2. At the

323 cut-off value of $\mathrm{r}=0.7$, three groups of intercorrelated variables were found and one

324 variable of each group was selected to continue with the analysis (i.e. CF, SED and SL).

325 In case the user would like to run the two-PCAs steps, both the first "screening PCA",

326 which is done over the uncorrelated variables, and the "final PCA" are subsequently

327 performed with the same function PCAs4clust(). In order to know the optimal number of

328 variables to be used in the "final PCA", a threshold of cumulative variance of the PCs is

329 implemented. This threshold is established to be 0.9 , i.e. $90 \%$ of the variance of the

330 variables explained, as default.

331 Finally, the clustering algorithm can be run over either the selected PCs or the

332 uncorrelated raw (phenology and productivity) variables using the function EFT_clust().

333 This function uses kmeans() from the package stats. K-means is an iterative unsupervised

334 method, one of the main limitations being that it is not able to optimize the number of

335 clusters by itself. Instead, the optimal number of clusters needs to be determined by the

336 user. In the $L P D y n R$ package, the optimal number of clusters can be determined using the

337 "scree-plot method". This method is implemented with the function clust_optim() and it

338 is based on running several K-means clustering with different number of clusters each, in

339 order to assess how the quality of the models change with the number of clusters. Then, a 
-This is a non-peer reviewed preprint submitted to EarthArXiv-

340 plot is produced with the number of clusters in the x-axis and the total within-cluster sum

341 of squares in the y-axis. A break line, the so-called "elbow", indicates the number of

342 clusters where the quality of the model no longer improves substantially as the number of

343 clusters (model complexity) increases. In the present study the clustering was run with

344 ten different number of clusters (5 to 50, with the increment of 5) to give a good amount

345 of points to plot the curve, and the maximum number of iterations was set to 10 (see the

346 plot produced in Supplementary Material Figure S3.1).

347 The "scree plot" method undoubtedly has some level of subjectivity, as the user decides

348 where the curve flattens enough for the appropriate number of clusters. Alternatively, to

349 remove such subjectivity, several numerical methods exist to calculate the optimal

350 number of clusters, although they take also some statistical assumptions. These methods

351 might be explored in the future if a higher level of accuracy is believed to be necessary or

352 if the process shall be performed without user intervention. In addition, other hierarchical

353 clustering methods could be explored in order to avoid calculating the optimal number of

354 clusters beforehand, although previous tests run with ISODATA have been shown to be

355 highly resource demanding, especially in terms of computing time.

356 Once the optimal number of clusters is estimated, the final clustering is run with the

357 function EFT_clust() using the defined number of clusters and passed with the argument

358 n_clust. Other parameters which can be passed to the function EFT_clust() are those that

359 will be passed to stats::kmeans(), such as nstart, iter.max or algorithm (see

360 https://stat.ethz.ch/R-manual/R-deve1/library/stats/html/kmeans.html for further

361 information). It is important to note that when setting the argument $n$ start, the larger the

362 value the more accurate the clustering result will be. This is because the function uses 
-This is a non-peer reviewed preprint submitted to EarthArXiv-

363 different sets of starting random centroids and runs the clustering nstart times. From

364 these number of clustering runs, the best classification result is chosen. Therefore, a

365 larger $n$ start value increases the chances of having a better cluster classification. In

366 addition, kmeans() can use different algorithms to perform the clustering (e.g.

367 "MacQueen", "Hartigan-Wong”, etc.; see references in kmeans() documentation). As

368 stated in the function documentation (?kmeans), "Hartigan-Wong" usually gives better

369 results, although it is recommended to try several starts ( start $>1$ ). However, when

370 using "Hartigan-Wong" with a (too) large number of clusters, and a lot of values of the

371 variables are very similar, kmeans() is not able to converge in an acceptable amount of

372 time (even increasing the number of iterations with iter.max). In these cases when the

373 clustering does not converge, instead of stopping the process with an error, the function

374 kmeans() only gives a warning after finishing the clustering, so that the obtained clusters

375 are based on a non-converged process. Diminishing the number of clusters or rounding

376 variables' values might be good strategies to help kmeans() to converge.

377 EFT_clust() produces a RasterLayer object, where each pixel is linked to a cluster, plus

378 an index of the clustering performance, which measures the compactness of individuals

379 (i.e. pixels) within the groups. This index, which is expected to be as high as possible, is

380 calculated as:

381

$$
C I=\frac{B S S}{T S S} \times 100
$$

382 where $C I$ is the compactness index, $B S S$ is between-cluster sum of squares (i.e.

383 betweenss, provided by kmeans()) and TSS is total sum of squares (i.e. totss, also

384 provided by kmeans()). 
-This is a non-peer reviewed preprint submitted to EarthArXiv-

385 Finally, as previous tests of K-means with up to 100 iterations were showing problems to

386 converge in a certain limit of time, the maximum number of iterations is set to 500 as

387 default in the function. Within this number of iterations and rounding variables, for

388 almost all the tests performed, the process did achieve convergence with no issues (see

389 Supplementary Material S4). For the running example, the EFTs resulted from the whole

390 process can be seen in Figure 3A.

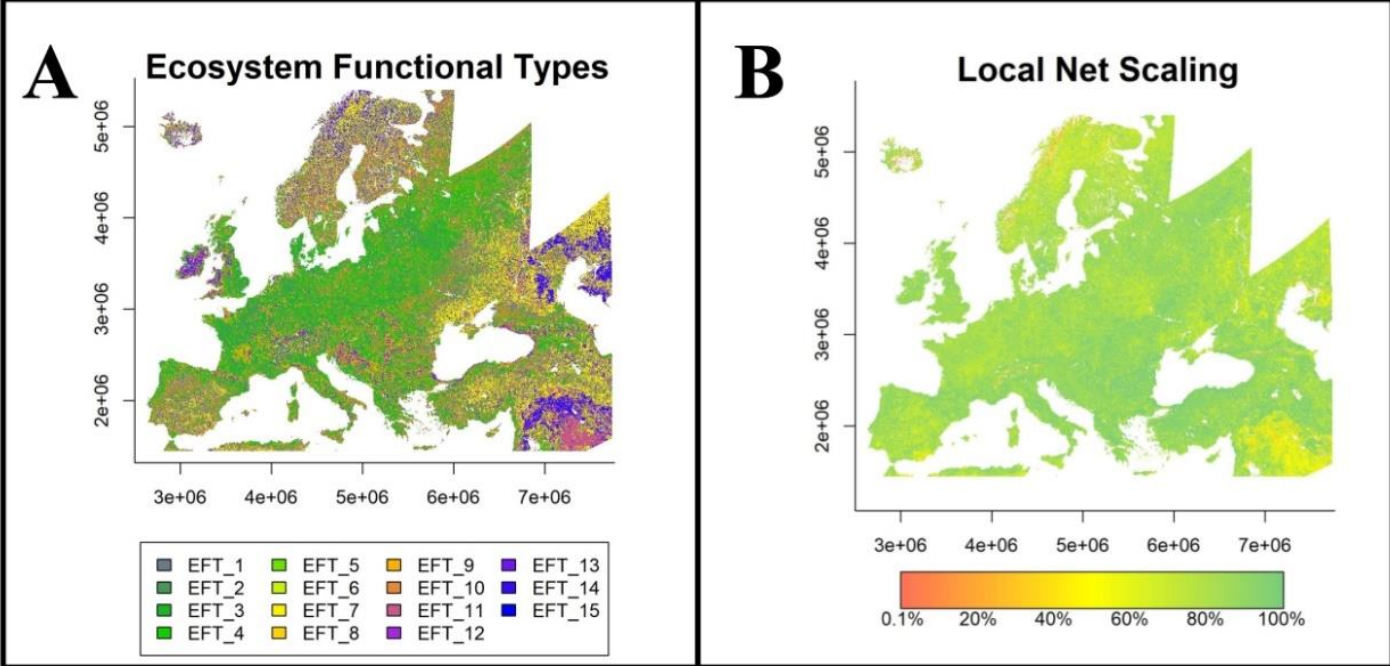

391

Figure 3: (A) Ecosystem Functional Types (EFTs) derived from phenological and productivity variables using the K-means clustering method. (B) Local Net Primary Production Scaling (LNS): proportion of annual production (i.e. average of the last 5 years of cyclic fraction) over the local potential production (i.e. the 90-percentile within the Ecosystem Functional Type)

\subsection{Local Net Production Scaling}

397 The Local Net Primary Productivity Scaling (from now on, Local Net Scaling or LNS)

398 method (Prince, 2009) is based on the use of multi-temporal satellite data to calculate the

399 difference between the potential and actual NPP for each pixel in homogeneous land 
-This is a non-peer reviewed preprint submitted to EarthArXiv-

400 functional units. Potential productivity in the LPDynR method is defined as the

401 productivity level which could be reached without human influence in natural landscapes

402 (Prince, 2009, and references therein) or as the result of human activity e.g. in agriculture

403 areas or managed forests, and is estimated as the maximum value of productivity within

404 each EFT. The deviation of the productivity found in a particular place and time as

405 referred to the local maximum within its phenological homogeneous cluster, reflects a

406 level of productivity anomaly which is useful for the productivity status map (Ivits and

407 Cherlet, 2013).

408 The cyclic fraction of vegetation productivity (e.g. the summed NDVI over the growing 409 season) is widely used as a proxy for the estimation of the current land productivity

410 (Fensholt, 2013), as it incorporates both natural and anthropogenic factors which define

411 the inter-annual variability of land production. Therefore, it represents that part of the

412 standing biomass which is potentially appropriated to be used by humans and the

413 environment (Ivits and Cherlet, 2013) and it is the one appropriated to calculate the LNS.

414 The function LNScaling() is implemented in LPDynR to calculate the LNS. The

415 productivity variable (i.e. CF) and the EFTs clusters as explained under 5.1 are passed to

416 LNScaling() to calculate the potential productivity within each EFT. Instead of the

417 maximum productivity value within each cluster, the 90-percentile value is established as

418 the potential productivity value, given that values higher than this threshold could be

419 outliers. Finally, the LNS for each pixel is calculated as

420

$$
L N S=\frac{A P}{P P_{E F T}}
$$


-This is a non-peer reviewed preprint submitted to EarthArXiv-

421 where $A P$ is the annual production of the pixel (i.e. the average of the last 5 years of

422 cyclic fraction) and $P P_{E F T}$ is the potential production within its EFT (i.e. the 90-

423 percentile).

424 For the calculation of the final LPD indicator (i.e. combined assessment), the Local Net

425 Scaling values are aggregated into two categories: (1) LNS pixels with less than $50 \%$ of

426 the potential local production (within the EFT) and (2) LNS pixels with more or equal to

$42750 \%$ of potential local production. This percentage, being $50 \%$ the default in LPDynR,

428 can be set by the user.

429 The result for the LNS calculation is presented in Figure 3B.

\section{Combined assessment of land productivity}

431 The Land Productivity Dynamics indicator, as shown in the processing flowchart in

432 Figure 1, is based on the combination of two main sources of information: a map of the

433 tendency, positive or negative, of the level of land productivity along the time series, and

434 another map capturing the current level of productivity of each pixel relative to the

435 maximum productivity in a homogeneous land area. As seen above, both branches to

436 calculate the indicator are qualitative methods. Therefore, the final LPD indicator,

437 produced with the function $L P D \_C o m b A s s e s s($ ), is also a qualitative measure with 5

438 possible values or categories after the reclassification of each pixel as shown in Table 3.

439 Such categories are (1) d - Declining, (2) ed - Early signs of decline, (3) st - Stable but

440 stressed, (4) sn - Stable and not stressed and (5) i - Increasing land productivity. 
-This is a non-peer reviewed preprint submitted to EarthArXiv-

441 Table 3: Lookup table for the combination of the two branches assessment (i.e. Long Term

442 Change Map and Current Status Map of land productivity) to derive the Land Productivity

443 Dynamics categories (i.e. (1) d-Declining land productivity, (2) ed-Early signs of decline of

444 land productivity, (3) st - Stable but stressed land productivity, (4) sn-Stable and not stressed

445 land productivity and (5) $i$ - Increasing land productivity). The Local Scaling is defined as 50\%

446

by default, but it can be modified by the user

\begin{tabular}{ccccc} 
Steadiness I. & Baseline L. & State Change & \multicolumn{2}{c}{ Local Scaling } \\
& & & $\mathbf{5 0 \%}$ & \rangle$=\mathbf{5 0 \%}$ \\
\hline st1 & lo & 0 & $\mathrm{~d}$ & ed \\
st1 & lo & 1 & $\mathrm{~d}$ & ed \\
st1 & lo & 2 & $\mathrm{~d}$ & $\mathrm{~d}$ \\
st1 & me & 0 & $\mathrm{~d}$ & ed \\
st1 & me & 1 & $\mathrm{~d}$ & ed \\
st1 & me & 2 & d & d \\
st1 & hi & 0 & ed & st \\
st1 & hi & 1 & d & ed \\
st1 & hi & 2 & d & ed \\
st2 & lo & 0 & st & st \\
st2 & me & 0 & st & st \\
st2 & hi & 0 & st & st \\
st3 & lo & 0 & sn & sn \\
st3 & me & 0 & sn & sn \\
st3 & hi & 0 & sn & sn \\
st4 & lo & 0 & sn & i \\
st4 & lo & 1 & sn & i \\
st4 & lo & 2 & i & i \\
st4 & me & 0 & sn & i \\
st4 & me & 1 & i & i \\
st4 & me & 2 & i & i \\
st4 & 0 & i & i
\end{tabular}

447

448 In the present study, the Land Productivity Dynamics indicator final map (Figure 4) is the

449 result of the combined assessment of the Long Term Change Map (Figure 2D) and the

450 Current Status Map of land productivity (Figure 3B), both based on the "Above ground 
-This is a non-peer reviewed preprint submitted to EarthArXiv-

451 vegetation productivity" variable, plus the two phenological variables for the derivation 452 of the EFTs.

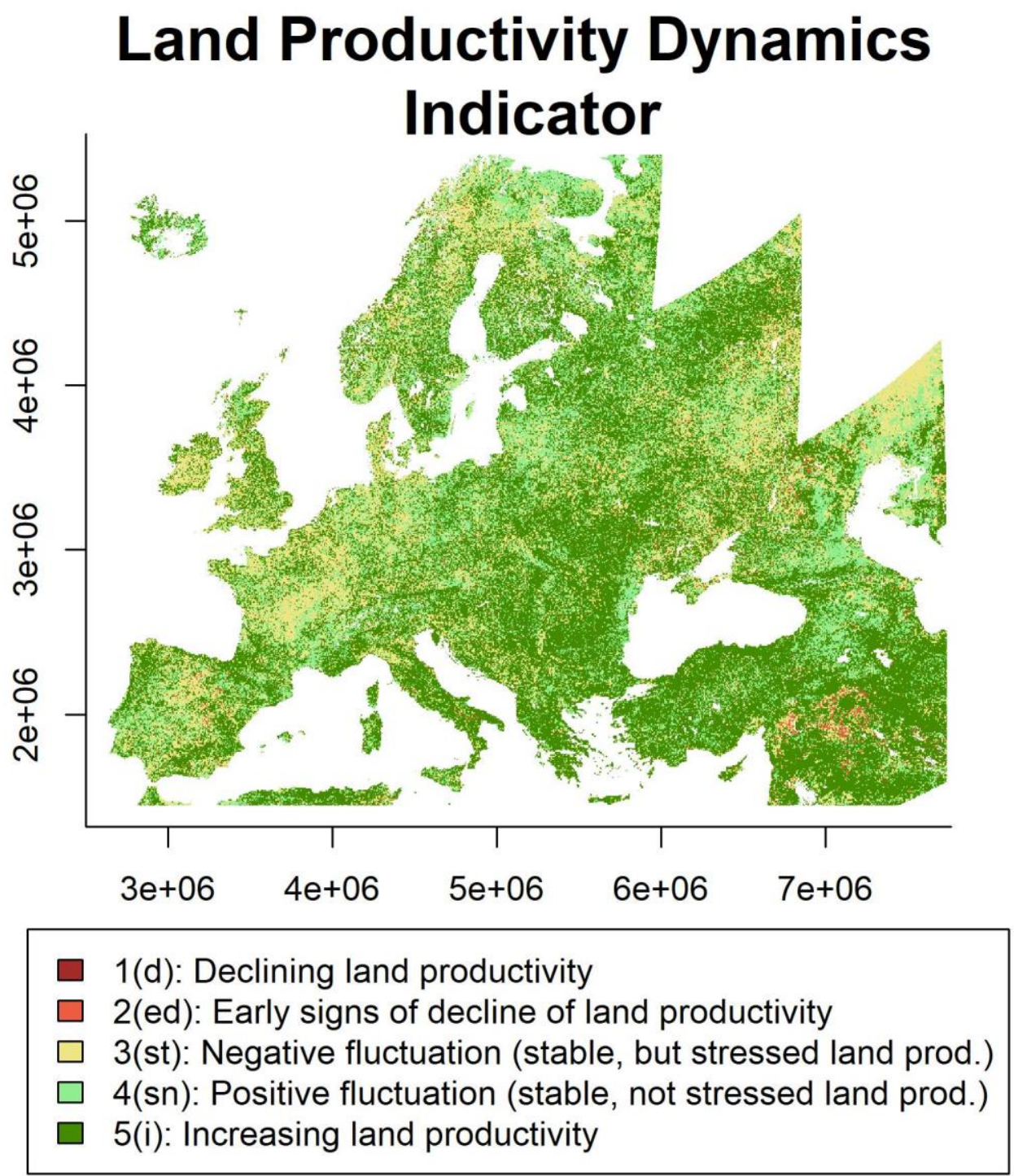

Figure 4: Land Productivity Dynamics indicator final map. Combined assessment of the Long productivity, (2) ed - Early signs of decline of land productivity, (3) st - Stable but stressed land productivity, (4) sn - Stable and not stressed land productivity and (5) $i$ - Increasing land 
-This is a non-peer reviewed preprint submitted to EarthArXiv-

460

461

462

463

464

465

466

467

468

469

470

471

472

473

474

475

476

477

478

\subsection{Alternative method for the Land Productivity Dynamics indicator}

Including the current level of land productivity relative to its potential (Chapter 5) in the final LPD calculation (Chapter 6) improves the land productivity indicator as LNS values may indicate not degradation in areas with a negative tendency of productivity, but where the level of productivity still remains high relative to other similar areas nearby. Despite this, the user might want to derive the final product based only on the tendency map (i.e. Long Term Change Map; Chapter 4), avoiding the inclusion of the Current Status Map derived with the Local Net Scaling approach. The function $L P D \_$CombAssess() performs this step by passing the argument LandProd_current $=N U L L$. By doing so, the function reclassifies the Long Term Change Map into the same 5 categories of the LPD indicator described above. Table 4 shows how the function executes the reclassification. 
-This is a non-peer reviewed preprint submitted to EarthArXiv-

480 Productivity Dynamics categories (i.e. (1) d - Declining land productivity, (2) ed - Early signs of 481 decline of land productivity, (3) st - Stable but stressed land productivity, (4) sn - Stable and not 482 stressed land productivity and (5) $i$ - Increasing land productivity)

\begin{tabular}{cccc} 
Steadiness I. & Baseline L. & State Change & LPD class \\
\hline st1 & lo & 0 & $\mathrm{~d}$ \\
st1 & lo & 1 & $\mathrm{~d}$ \\
st1 & lo & 2 & $\mathrm{~d}$ \\
st1 & me & 0 & $\mathrm{~d}$ \\
st1 & me & 1 & $\mathrm{~d}$ \\
st1 & me & 2 & $\mathrm{~d}$ \\
st1 & hi & 0 & ed \\
st1 & hi & 1 & $\mathrm{~d}$ \\
st1 & hi & 2 & $\mathrm{~d}$ \\
st2 & lo & 0 & st \\
st2 & me & 0 & st \\
st2 & hi & 0 & st \\
st3 & lo & 0 & sn \\
st3 & me & 0 & sn \\
st3 & hi & 0 & sn \\
st4 & lo & 0 & sn \\
st4 & lo & 1 & sn \\
st4 & lo & 2 & i \\
st4 & me & 0 & sn \\
st4 & me & 1 & i \\
st4 & me & 2 & i \\
st4 & hi & 0 &
\end{tabular}

483

484 A comparison of the final LPD indicator map produced using the combined assessment 485 (i.e. Long Term Change Map + Current Status Map) with the one developed without the 486 Current Status Map can be seen in Figure 5 (Map 1 and Map 2, respectively). In addition, 487 the "differences map" in the same figure represents pixels which have a different class 488 between the two approaches. The difference between the classes was always equal to 
-This is a non-peer reviewed preprint submitted to EarthArXiv-

489 minus 1, indicating that the difference between the two approaches is only one class.

490 Furthermore, the combined indicator using the LNS approach had higher values in all

491 cases indicating a better potential to differentiate between land productivity conditions.

492 Table 5 shows the number of pixels which changed from one class to another. From this

493 table it can be seen how pixels never changed from negative to positive dynamics (class 3

494 to 4 ) or from positive to negative (class 4 to 3 ).

\section{Comparison LPD final maps produced with Combined Assessment and with reclassification of Long Term Change Map}
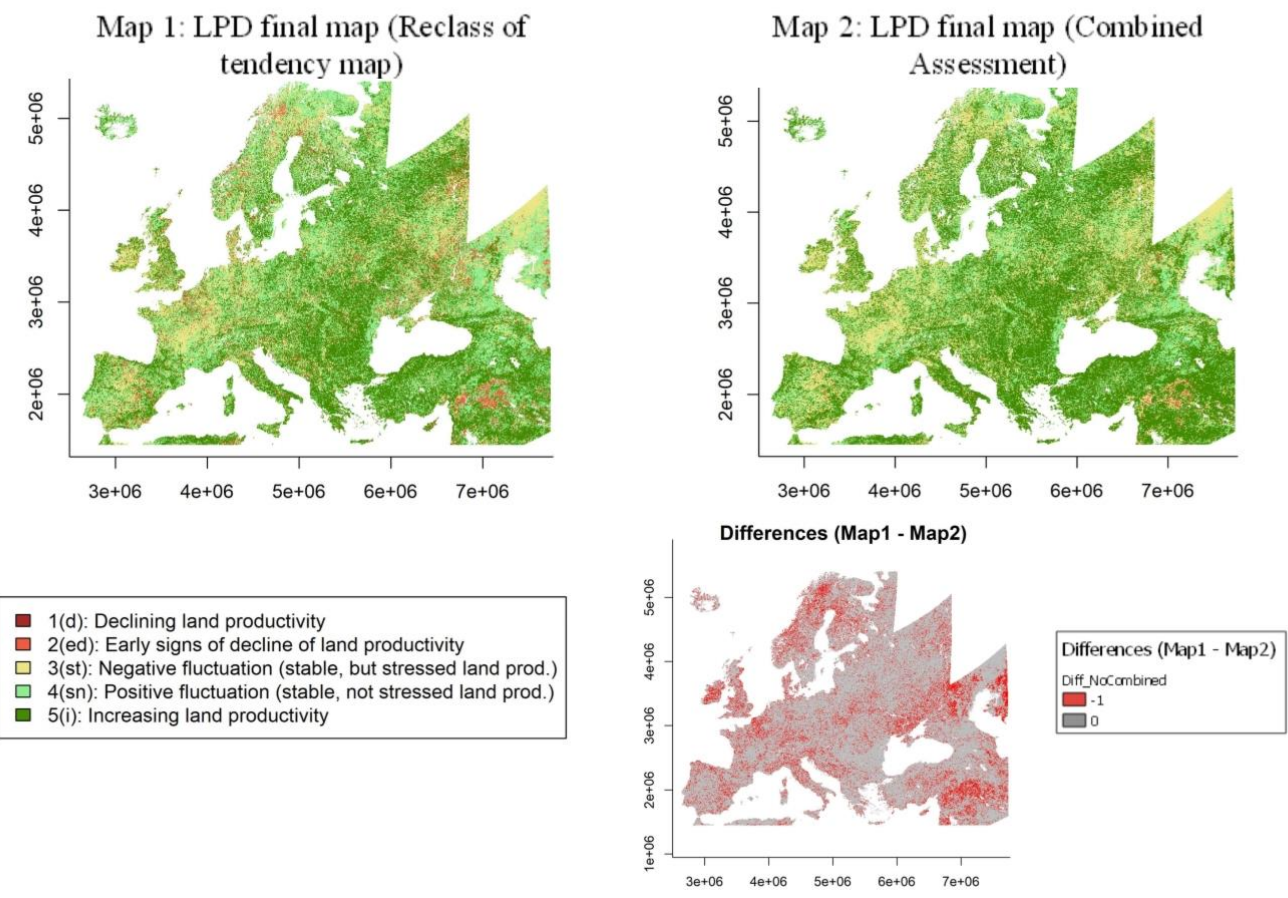

496 Figure 5: Land Productivity Dynamics indicator final maps derived by the reclassification of the

497 Long Term Change Map of land productivity (Map 1) and produced by the combined assessment

498 (Map 2; Long Term Change Map + Current Status Map). Differences Map (Map 1 - Map2)

499 represents in red those pixels showing different resulting classes from both approaches 
-This is a non-peer reviewed preprint submitted to EarthArXiv-

501

502

503

Non-combined
Assessment - Class

1

2

4

5 combinations were found in the case study

504

505 Finally, Figure 6 shows the proportion of pixels per LPD class under each approach, both

506 for the whole extent (i.e. Europe) and also splitting the map by biogeographical regions.

507 The biogeographical regions were defined with the official delineations used in the

508 Habitats Directive (92/43/EEC) and for the EMERALD Network, which are freely

509 distributed as a spatial data set by the European Environmental Agency - European

510 Commission (https://www.eea.europa.eu/data-and-maps/figures/biogeographical-and-

511 marine-regions-in).

512 The plots show that there were some differences in the proportion of pixels per class for

513 each of the two approaches. For example, the Alpine, the Anatolian, and the Steppic

514 regions were the three showing more differences, which ranged from 12.1 to $15.5 \%$ for

515 some LPD classes. This fact evidences the added value of including the Current Status

516 Map in the calculations to refine the LPD indicator final results. 
-This is a non-peer reviewed preprint submitted to EarthArXiv-

\section{Comparison LPD Methods by Bio-Geographical Regions (Combined Assessment vs LongTermChangeM Reclassification)}

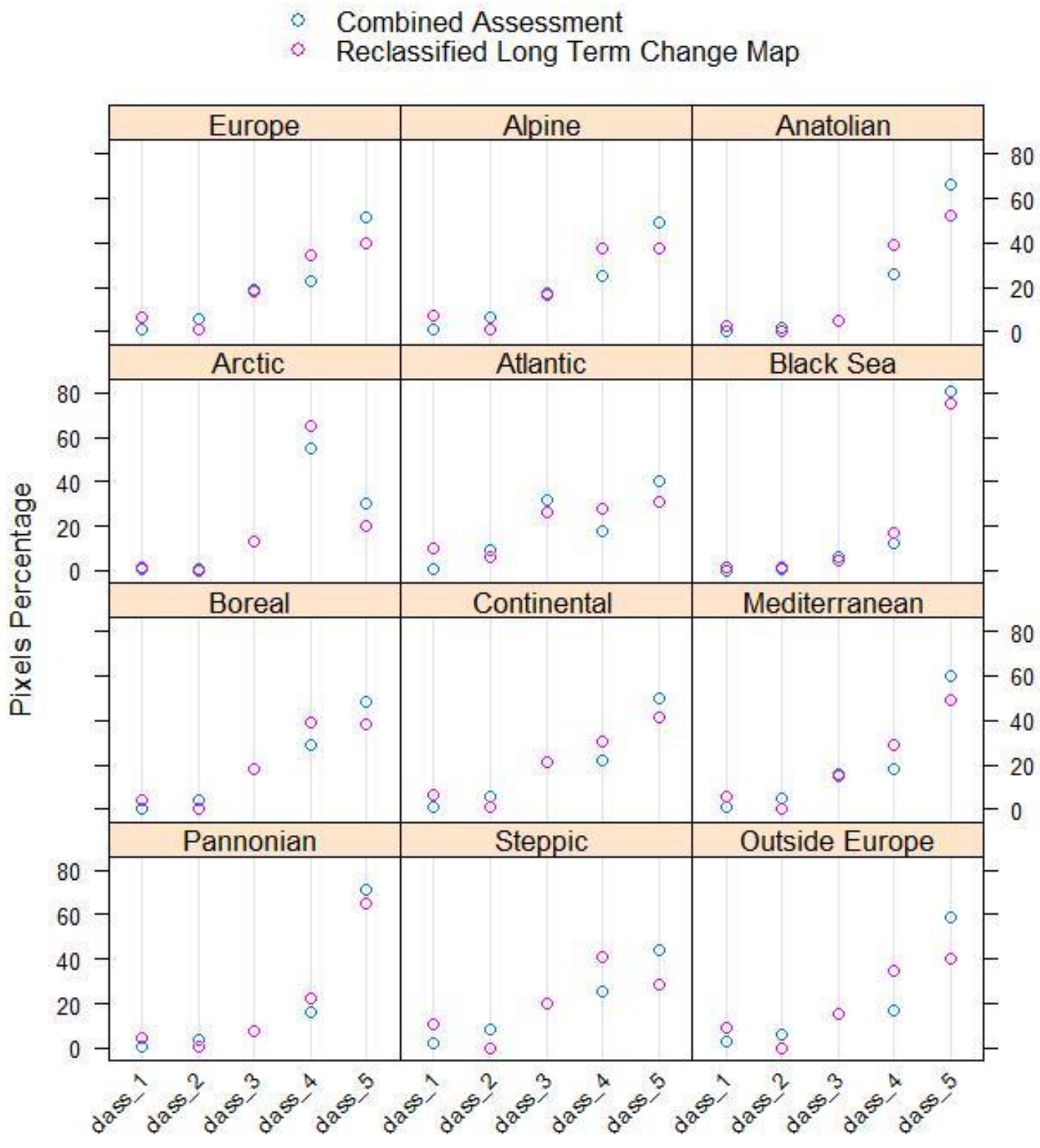

518

LPD Class

Figure 6: Proportion of pixels per LPD class for the combined assessment (light blue) and for the reclassified Long Term Change Map (purple), for Europe and by biogeographical regions 
-This is a non-peer reviewed preprint submitted to EarthArXiv-

\section{6.2 Land Productivity Dynamics partial indicator}

523 As seen in the previous subsections regarding the derivation of the tendency map (i.e.

524 Long Term Change Map; Chapter 4), the final result is related to the extremes of the time

525 series. In case the time series is long, the LPD indicator shows a long term assessment of

526 what has happened regarding the land productivity dynamics between the beginning and

527 the end of the period in the study. However, to understand the dynamics of the biomass

528 within the observation period, as well as to assess the stability of the final product, it

529 might be useful to produce several "partial LPD indicators" using different time windows

530 of the time series.

531 This process is not yet implemented in LPDynR as a function, but we propose the

532 following code to produce partial LPD maps of $n$ years and with an overlap of $y$ years

533 between the end of the last period and the beginning of the next one. This example was

534 implemented for the same case study shown along this article and the final partial LPD

535 maps can be seen in Figure 7.

536

537

538

539

540

541 
-This is a non-peer reviewed preprint submitted to EarthArXiv-

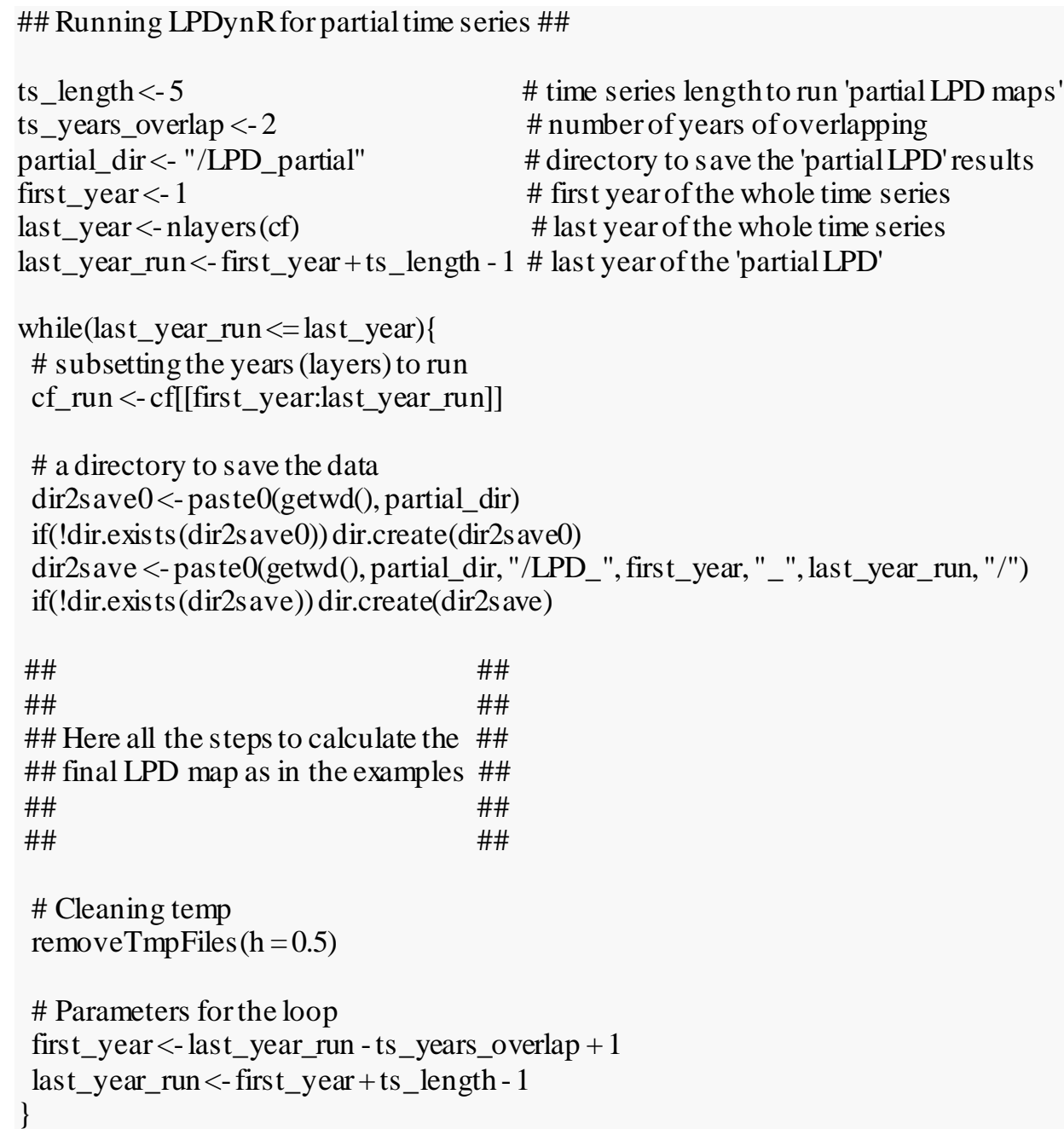




\section{Partial LPD Indicators}

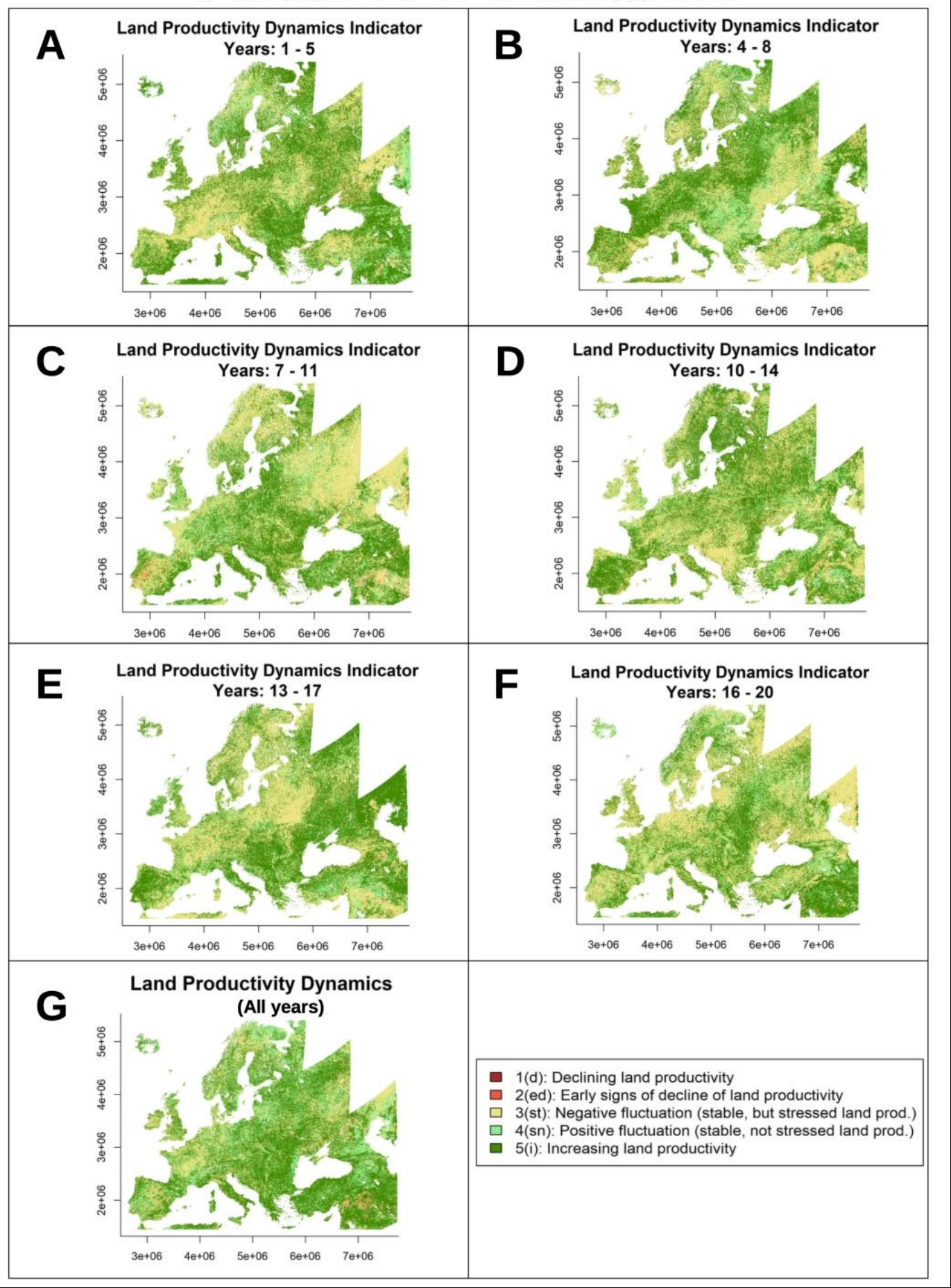

577 Figure 7: Partial LPD indicators (plots A to F) and LPD indicator for the whole time series (plot

$578 G$ ). The partial LPD indicators were produced for time windows of 5 years with an overlap of 2 
-This is a non-peer reviewed preprint submitted to EarthArXiv-

580 The complete LPD indicator (i.e. for the whole time series; Figure 7G) shows, in general

581 terms, a positive trend pattern across Europe (i.e. more pixels in greens). However, some

582 of the intermediate plots show more negative trends (i.e. yellow and light red pixels).

583 This, besides demonstrating the highly fluctuating character of vegetation, confirms the

584 influence of the extremes of the time series on the final result. In this sense, in the time

585 series of the example, the first period seemed to show stressed vegetation in terms of

586 productivity for most of the pixels in Western/Central Europe, and they expressed a large

587 increase around years 7/8. Such increase caused a large number of areas belonging to the

588 higher LPD class, and it still influenced the dynamics of the following period, resulting in

589 areas with stressed vegetation.

590 The fact that the LPD indicator calculated with the approach included in LPDynR is

591 influenced by the beginning and the end of the time series is not a limitation, as the main

592 goal of the LPD indicator is to know the current state of vegetation in relation to a

593 previous state, and not the fluctuations due to, for example, to extreme climatic events

594 such as e.g. droughts. However, being able to map these fluctuations in space and time

595 might add information for further analysis.

\section{$596 \quad 7$ Conclusions}

597 As stated by the Intergovernmental Science-Policy Platform on Biodiversity and

598 Ecosystem Services (IPBES), land degradation leads to a loss of biodiversity and a

599 reduction of ecosystem functions and delivered services all over the world. Therefore,

600 combating land degradation and restoring degraded lands has become an urgent priority

601 in order to protect all life on Earth as well as to ensure human well-being (IPBES, 2018). 
-This is a non-peer reviewed preprint submitted to EarthArXiv-

602 In this sense, satellite observations provide valuable data which might help to monitor the

603 Earth's land cover to evaluate the state of land degradation.

604 The Land Productivity Dynamics indicator (LPD), as part of the SDG-15.3.1 indicator,

605 aims at contributing to the assessment of the state of land degradation and desertification

606 at global, regional and local scales. Therefore, the LPDynR new tool has been developed

607 to derive the LPD indicator using phenological and land productivity variables, which

608 can be obtained from long-term time series of Earth observation imagery.

609 LPDynR is a comprehensive set of open source programming code, written in the well-

610 known $\mathrm{R}$ language and properly packaged, ready to be freely distributed in order to let

611 the users with a minimum knowledge of the R language calculate the LPD indicator. The

612 package, once installed, includes several examples and a small data set for testing the

613 functionalities and the different parameters to tune them.

614

615

6168 References

617 Cherlet, M., Hutchinson, C., Reynolds, J., Hill, J., Sommer, S., Maltitz, G. von (Eds.),

618 2018. World atlas of desertification. Publication Office of the European Union,

619 Luxembourg. 
-This is a non-peer reviewed preprint submitted to EarthArXiv-

620 Dubovyk, O., 2017. The role of remote sensing in land degradation assessments:

621 Opportunities and challenges. European Journal of Remote Sensing 50, 601-613.

622 https://doi.org/10.1080/22797254.2017.1378926

623 FAO, 2019. Trees, forests and land use in drylands: The first global assessment (FAO $624 \quad$ Forestry Paper No. 184). FAO, Rome.

625 Fensholt, K.K., Rasmus Rasmussen, 2013. Assessing land degradation/recovery in the 626 african sahel from long-term earth observation based primary productivity and 627 precipitation relationships. REMOTE SENSING 5, 664-686.

628 Guo, W.Q., Yang, T.B., Dai, J.G., Shi, L., Lu, Z.Y., 2008. Vegetation cover changes and 629 their relationship to climate variation in the source region of the yellow river, china,

632 IPBES, 2018. The ipbes assessment report on land degradation and restoration.

633 Montanarella, 1., scholes, r., and brainich, a. (Eds.). Secretariat of the

634 Intergovernmental Science-Policy Platform on Biodiversity; Ecosystem Services, 635 Bonn, Germany.

636 Ivits, E., Cherlet, M., 2013. Land-productivity dynamics towards integrated assessment 637 of land degradation at global scales (Technical Report No. EUR 26052). Joint 638 Research Centre of the European Commission.

639 Ivits, E., Cherlet, M., Horion, S., Fensholt, R., 2013. Global biogeographical pattern of 640 ecosystem functional types derived from earth observation data. Remote Sensing 5, 641 3305-3330. https://doi.org/10.3390/rs5073305 
-This is a non-peer reviewed preprint submitted to EarthArXiv-

642 Ivits, E., Cherlet, M., Mehl, W., Sommer, S., 2013. Ecosystem functional units

643 characterized by satellite observed phenology and productivity gradients: A case

644 study for europe. Ecological Indicators 27, 17-28.

645 https://doi.org/10.1016/j.ecolind.2012.11.010

646 Ivits, E., Cherlet, M., Sommer, S., Mehl, W., 2013. Addressing the complexity in non-

647 linear evolution of vegetation phenological change with time-series of remote

648 sensing images. Ecological Indicators 26, 49-60.

649 https $/ / /$ doi.org/https $/ / /$ doi.org/10.1016/j.ecolind.2012.10.012

650 Jin, H., Eklundh, L., 2014. A physically based vegetation index for improved monitoring

651 of plant phenology. Remote Sensing of Environment 152, 512-525.

652 https://doi.org/https ://doi.org/10.1016/j.rse.2014.07.010

653 Jönsson, P., Eklundh, L., 2004. TIMESAT — a program for analyzing time-series of 654 satellite sensor data. Computers \& Geosciences 30, 833-845.

655 Leroy B., Meynard C.N., Bellard C., Courchamp F., 2016. virtualspecies, an R package 656 to generate virtual species distributions. Ecography 39, 599-607.

657 https://doi.org/10.1111/ecog.01388

658 Middleton, N., Stringer, L., Goudie, A., Thomas, D., 2011. The forgotten billion. MDG 659 achievement in the drylands. United Nations Development Programme, New York, $660 \quad$ NY, 10017, USA.

661 Orr, B.J., Cowie, A.L., Castillo Sanchez, V.M., Chasek, P., Crossman, N.D., Erlewein, 662 A., Louwagie, G., Maron, M., Metternicht, G.I., Minelli, S., Tengberg, W., A. E., 
-This is a non-peer reviewed preprint submitted to EarthArXiv-

663 Welton, S., 2017. Scientific conceptual framework for land degradation neutrality.

664 In: A report of the science-policy interface. UNCCD, Bonn, Germany.

665 Prince, I.R., S. D. Becker-Reshef, 2009. Detection and mapping of long-term land

666 degradation using local net production scaling: Application to zimbabwe.

$667 \quad$ REMOTE SENSING OF ENVIRONMENT 113, 1046-1057.

668 Sims, N.C., Barger, N.N., Metternicht, G.I., England, J.R., 2020. A land degradation

669 interpretation matrix for reporting on un sdg indicator 15.3.1 and land degradation

670 neutrality. Environmental Science \& Policy 114, 1-6.

671 https://doi.org/https $: / /$ doi.org/10.1016/j.envsci.2020.07.015

672 Sims, N.C., Green, C., Newnham, G.J., England, J.R., Held, A., Wulder, M.A., al., 2017.

673 Good practice guidance sdg indicator 15.3.1. Proportion of land that is degraded

674 over total land area, First. ed. United Nations Convention to Combat

$675 \quad$ Desertification.

676 UN, 2015. Transforming our world: The 2030 agenda for sustainable development (No.

677 A/RES/70/1). United Nations.

678 UNCCD, 2015. Integration of the sustainable development goals and targets into the

679 implementation of the United Nations convention to combat desertification and the

680 report of the intergovernmental working group on land degradation neutrality (No.

681 ICCD/COP(12)/4). UNCCD Conference of the Parties, Ankara, Turkey.

682 Yengoh, G.T., Dent, D., Olsson, L., Tengberg, A.E., Tucker, C.J., 2015. Use of the

683 normalized difference vegetation index (ndvi) to assess land degradation at multiple

684 scales, Springer briefs in environmental science. Springer. 